\title{
Prescription of pharmacotherapy for depression in elderly people varies with age, race, gender, and length of care
}

Strothers HS, Rust G, Minor P, et al. Disparities in antidepressant treatment in Medicaid elderly diagnosed with depression. J Am Geriatr Soc 2005;53:456-61.

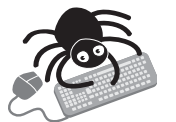

This article

contains extra text on the EBMH website

\section{What is the prevalence of drug treatment for depression in different populations of elderly people insured by Medicaid?}

\section{METHODS}

1

Design: Cross sectional study.

(3)

Setting: Medicaid claims data from five US states in 1998

으 Population: 7339 people aged 65-84 years with an outpatient Medicaid claim for depression (international classification of diseases diagnosis). Exclusions: bipolar illness or dysthymia.

Assessment: Information about Medicaid recipients, including demographics, final action, paid claims, and days in long term care, was obtained from insurance claims data. National drug codes were used to identify prescriptions for antidepressants. Rates of drug treatment were calculated with $95 \%$ confidence intervals. Logistic regression was used to assess the effect of multiple factors on drug treatment.

F(n)

Outcomes: Prevalence of antidepressant prescriptions.

\section{MAIN RESULTS}

In elderly people with depression, $24 \%$ received no antidepressant treatment, $26.3 \%$ received old antidepressants alone or in combination, $64.3 \%$ received new antidepressants alone or in combination, $14.8 \%$ received both new and old antidepressants, and $75.8 \%$ were treated with any antidepressant (old, new, or both) in the same calendar year. Thirty seven per cent of black people, $22.4 \%$ of white people, 13.8\% of Asian people and 23.6\% of Hispanic people received no drug treatment for depression. Significantly more black, nonHispanics were receiving no treatment than whites $(\mathrm{p}<0.001)$. Other risk factors for no pharmacotherapy were being male, aged 65-74 years and being in long term care (see http://www.ebmentalhealth.com/supplemental for table).

\section{CONCLUSIONS}

In the USA, the number of elderly people insured by Medicaid and not receiving drug treatment for their depression is high. Being male, black non-Hispanic, in long term care, and aged 65-74 years old are risk factors for lack of drug treatment.

\section{NOTES}

In this study, "old" antidepressants included tricyclics and monoamine oxidase inhibitors, and "new" antidepressants were selective serotonin reuptake inhibitors. The study found that State had a significant effect on no drug prescription and on prescription of new antidepressants. State practice may be a confounder in this study. Prevalence studies do not explore causal links, and discrepancies in

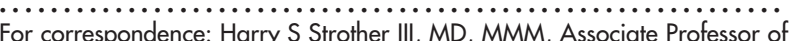
Family Medicine and Geriatric Team Leader, National Center for Primary Care, Morehouse School of Medicine, 1513 E Cleveland Avenue, Building \# 100, Suite 300-A, East Point, GA 30344, USA; Strothh@msm.edu

Sources of funding: Health Care Financing Administration, Centers for Medicare and Medicaid Services Contract, National Institutes of Health National Center on Minority Health, and Health Disparities Grant. treatment rates may be due to patient, provider, or system level practice.

\section{Commentary}

M

any studies have demonstrated disparities in depression treatment, such as by ethnicity, income, education, geography, age, and gender. However, the role of culture, economics, insurance, and other factors in these disparities is poorly understood. Studies in the US Medicaid population are of interest as benefits are, at least formally, the same across subgroups, and differences in economic resources in these means tested programmes are smaller than in general population samples. Insurance disparities clearly play an important role, but persistent differences in Medicaid treatment patterns, apparent across a range of diseases, ${ }^{12}$ call attention to non-economic factors. Strothers et al provide the most recent of a number of studies documenting disparate treatment patterns for depression among the elderly. As with earlier studies, 34 they have found lower rates of antidepressant treatment among African-American elderly diagnosed with depression.

Such findings raise important practice and policy issues. Despite research documenting disparities, much more work is needed to clarify the processes that cause them, and hence the most promising targets for policy intervention. Two ancillary findings by Strothers et al are suggestive in this regard. First, non-Hispanic whites were almost three times as likely as African-Americans to have a depression diagnosis in claims over a one year period. Disparities in depression recognition, as well as in treatment rates once identified, are clearly important. Better research is also needed on underlying depression rates and symptomatology patterns across racial-ethnic subgroups of elderly (the literature is somewhat inconsistent on this issue). Second, substantial treatment differences were found across the five states examined, with nontreatment rates in Arkansas almost twice as high as in Washington. It would be interesting to examine whether disparities are greater in states with lower overall treatment rates. Such differences also highlight the importance of monitoring the impact of transition to new Medicare drug plans that will take place in 2006 for most elderly Medicaid beneficiaries. It is possible that the transition to a federally administered system will reduce regional disparities, but it will also be essential to monitor the impact of features of the new system, such as its complexity, new copayments, and formulary requirements on access and disparities. ${ }^{5}$

Stephen Crystal, PhD

Director, Center for Health Services Research on Pharmacotherapy, Chronic Disease Management, and Outcomes, Institute for Health, Health Care Policy, and Aging Research, Rutgers University, New Brunswick,

1 Schore J, Brown R, Lavin B. Racial disparities in prescription drug use among dually eligible beneficiaries. Health Care Financ Rev 2003;25:77-90.

2 Crystal S, Sambamoorthi U, Moynihan P, et al. Initiation and continuation of newer antiretroviral treatments among Medicaid recipients with AIDS. $J$ Gen Internl Med 2001;16:850-9.

3 Melfi CA, Croghan TW, Hanna MP, et al. Racial variation in antidepressant treatment in a Medicaid population. J Clin Psychiatry 2000;61:16-21.

4 Crystal S, Sambamoorthi U, Walkup J, et al. Diagnosis and treatment of depression in the elderly Medicare population: predictors, disparities and trends. J Am Geriatr Soc 2003;51:1718-21.

5 Kaiser Commission on Medicaid and the Uninsured. The new Medicare prescription drug law: issues for enrolling dual eligibles into drug plans. Washington: Kaiser Family Foundation, 2005. Available at www.kff.org/ medicare/upload/The-New-Medicare-Prescription-Drug-Law-Issue-forEnrolling-Dual-Eligibles-into-Drug-Plans-Issue-Paper.pdf (accessed September 2005) 